\title{
Precisión en la determinación de glucosa, colesterol y triglicéridos séricos, en laboratorios clínicos de Lima, Perú
}

Accuracy in determining serum glucose, cholesterol and triglycerides levels in clinical laboratories of Lima, Peru

\author{
Miguel H. Sandoval Vegas ${ }^{1}$, Heli J. Barrón Pastor ${ }^{1}$, Rudi A. Loli Ponce ${ }^{1}$, \\ Yvan V. Salazar Criado ${ }^{2}$ \\ Centro de Investigación de Bioquímica y Nutrición, Facultad de Medicina, Universidad Nacional Mayor de San Marcos. \\ ${ }^{2}$ Medical Bussines SAC
}

\begin{abstract}
Resumen
Objetivos: Evaluar la precisión de laboratorios de análisis clínicos de Lima, en la determinación de glucosa, colesterol y triglicéridos séricos. Diseño: Descriptivo. Lugar: Centro de Investigación de Bioquímica y Nutrición, Facultad de Medicina, UNMSM, y laboratorios clínicos participantes de Lima. Participantes: Muestras séricas de donantes. Intervenciones: Previo consentimiento informado, se envió muestras séricas ciegas duplicadas a 88 laboratorios clínicos, que constituyeron la muestra; el traslado de los sueros fue en cadena de frío de 4 a $6^{\circ} \mathrm{C}$. Los resultados fueron recibidos vía correo electrónico y con ellos se obtuvo la media, desviación estándar $(\mathrm{DE})$, coeficiente de variación (CV) y el índice de desviación estándar (SDI); también se valoró la precisión usando la validación de la variabilidad biológica (VB). Principales medidas de resultados: Concentración de glucosa, colesterol y triglicéridos. Resultados: La mayoria (>75\%) de los resultados de los laboratorios se encontraron dentro del rango aceptable; hubo laboratorios fuera del rango de control, entre 9,1 a 12,5\% de ellos. La evaluación del índice de calidad mediante la variabilidad biológica para la mayoría de laboratorios estuvo en control, sea esta óptima, deseable o mínima; $42 \%$ de los laboratorios estuvo fuera de control para la prueba del colesterol, $25 \%$ fuera de control para la glucosa y 11,4\% para triglicéridos. Los laboratorios con equipos automatizados presentaron mejor precisión. Conclusiones: Los laboratorios clínicos en su mayoria tuvieron buena precisión en las mediciones; sin embargo, aún existen laboratorios con amplia imprecisión en sus resultados, por lo que deben hacerse esfuerzos para mejorar estos índices de calidad.

Palabras clave: Laboratorios, control de calidad, variaciones dependientes de métodos y procedimientos.

\section{Abstract}

Objectives: To assess the accuracy of clinical laboratories in Lima in the determination of glucose, cholesterol and triglycerides. Design: Descriptive. Location: Center for Research in Biochemistry and Nutrition, Faculty of Medicine, San Marcos University, and clinical laboratories participating in Lima. Materials: Serum samples from donors. Interventions: Prior informed consent, serum samples were sent in $4-6^{\circ} \mathrm{C}$ cold chain to 88 blind duplicate clinical laboratories sampled. Results were received via email and arithmetic mean, standard deviation (SD), coefficient of variation (CV) and standard deviation index (SDI) were obtained; accuracy was assessed using the validation of biological variability (BV). Main outcome measures: Glucose, cholesterol and triglycerides levels. Results: The majority $(>75 \%)$ of laboratory results were within acceptable range; laboratories out of control range were 9,1 to $12,5 \%$. Quality index by biological variability for most laboratories was in control, whether optimal, desirable or minimum; $42 \%$ of the laboratories were found out of control for cholesterol testing, $25 \%$ for glucose, and $11.4 \%$ for triglycerides. Laboratories with automated equipment had better accuracy. Conclusions: Most clinical laboratories had good measurements accuracy; however, there were several laboratories with extensive vagueness in their results where efforts should be done to improve these quality indices.
\end{abstract}

Key words: Laboratories, quality control, methods and procedures variations.

An Fac med. 2012;73(3):233-8

\section{INTRODUCCIÓN}

En el Perú, como en el mundo entero la medicina tiene entre sus soportes la ayuda diagnóstica del laboratorio y de la imagenología, las cuales brindan al clínico información valiosa del estado del paciente y con ello el respaldo para la toma de decisiones médicas en el tratamiento de las diversas patologías. Los resultados de los estudios de pruebas auxiliares son cada vez más exactas y su grado de asociación y capacidad diagnóstica son también más acertados. Sin embargo, es necesario que dichas pruebas sean convenientemente ejecutadas y controladas. Existe cada vez mayor conocimiento y aplicación del control de calidad como una necesidad en las actividades del laboratorio, un modo de excelencia para el trabajo y un procedimiento requerido para la validación de los resultados, tanto con el control interno como externo, a fin de garantizar la confiabilidad de los resultados; los controles básicos usados como indicadores usualmente utilizados son la precisión y la exactitud ${ }^{(1,2)}$.
Existen en la actualidad programas internacionales de control externo de la calidad para evaluar los resultados de los laboratorios ${ }^{(3,4)}$ : En nuestro medio son muy pocos los laboratorios de análisis clínicos que pueden evidenciar estos procesos de calidad, tal vez por los costos elevados o por la envergadura del establecimiento. Es una máxima del control de calidad que este deba iniciarse en el mismo laboratorio y luego con otros establecimientos, de tal manera que se pueda establecer la semejanza, confiabilidad y exactitud de los 
resultados del laboratorio clínico ${ }^{(5,6)}$. En el Perú, recientemente el Ministerio de Salud ha publicado la Norma Técnica NT 072, aprobada por resolución ministerial № 627-2008/ MINSA, en la que se establece la necesidad y obligatoriedad de establecer los mecanismos de control de la calidad como un medio en la mejora de la atención asistencial ${ }^{(7)}$.

En el control de la calidad se observa diversas etapas y mecanismos de ejecución. Sea en los procesos administrativos o procedimentales, en el laboratorio clínico existen dos procesos básicos de control: la precisión y la exactitud. La precisión intralaboratorio es definida como la concordancia observada entre los resultados obtenidos, al analizar varias veces una misma muestra en el mismo laboratorio; la mejor forma de conocerla es mediante pruebas de repetibilidad y de control de calidad interno ${ }^{(7,8)}$. Lo que se cuantifica en el laboratorio es la imprecisión, y esta es expresada generalmente en términos de la desviación estándar (DE). Como la $\mathrm{DE}$ depende de la concentración de la sustancia en el material para control, a veces es conveniente expresar la imprecisión en términos del coeficiente de variación $(\mathrm{CV})$, el cual expresa el valor porcentual de la desviación estándar con respecto al promedio de los valores obtenidos ${ }^{(8,9)}$.

Usualmente, los laboratorios determinan que existe un error cuando un resultado excede el promedio $(\mathrm{X}) \pm 2$ $\mathrm{DE}$, lo cual no es válido cuando las DE son amplias. Por lo tanto, es necesario verificar, antes de aplicar las reglas de control de calidad interno, que las DE y CV obtenidos no sobrepasen los límites de variabilidad máxima permisible. Estos límites pueden ser establecidos básicamente mediante tres métodos: criterios clínicos, variabilidad biológica y precisión real obtenida en la práctica (estado del arte). Según este último criterio, la variación máxima permisible la constituye el coeficiente de variación promedio obtenido por un grupo representativo de laboratorios en encuestas de variabilidad intralaboratorio ${ }^{(7,10)}$.

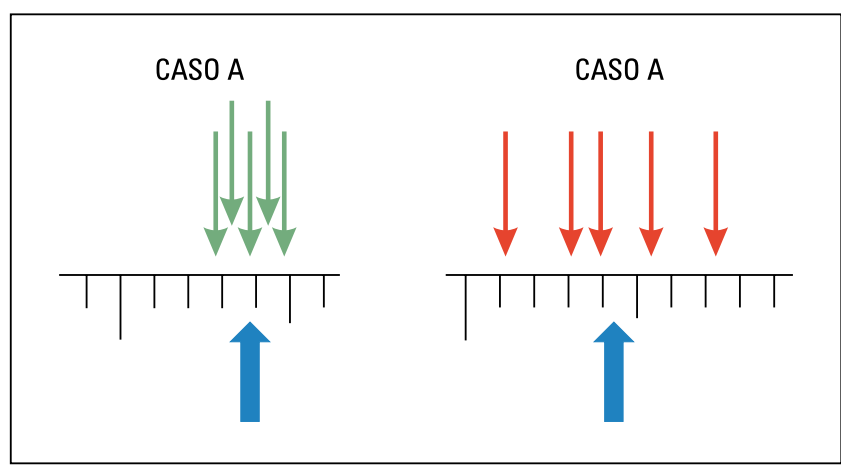

Figura 1. Se muestra la precisión de dos casos de mediciones repetidas (explicación en el texto).

La precisión es la expresión del grado de dispersión de las mediciones. Pero, como en la labor metrológica del laboratorio se realiza una medición de un analito particular en la muestra de un paciente, la precisión se convierte en una probabilidad, puesto que no se mide dos o más veces el mismo analito de la misma muestra. En la figura 1 se observa mediciones repetidas del analito de una misma muestra realizadas por dos laboratorios o dos operadores diferentes o por dos equipos o aparatos o metodologías diferentes de la misma muestra. Se observa fácilmente que el caso $\mathrm{B}$ tiene datos más dispersos que el caso A; por lo tanto, mayor DE y mayor CV porcentual; y como el resultado final es el que muestra la variación u oscilación, esta es la suma del total de variaciones u oscilaciones involucradas como equipos, aparatos, fluido eléctrico, temperatura ambiente, entre otros. Sin embargo, las mediciones deben ser realizadas en las mismas condiciones, para así se puedan circunscribir a menos variables intervinientes.

Nótese también en la figura 1 las flechas azules, que corresponden a las medias o promedios de las mediciones para los casos A y B, respectivamente, y según la escala estas pueden ser muy cercanas o coincidentes. Pero, por sí solo este valor (el promedio) no denota el grado de imprecisión y mucho menos el de exactitud, término que tiene otra connotación.

El objetivo de esta investigación, dentro del proyecto de calidad metrológica desarrollado en el Centro de In- vestigación de Bioquímica y Nutrición de la Facultad de Medicina de la Universidad Nacional Mayor de San Marcos, fue determinar la precisión en las determinaciones de glucosa, colesterol y triglicéridos séricos en laboratorios clínicos de Lima Metropolitana, que voluntariamente desearon participar.

\section{MÉTODOS}

Para este estudio descriptivo, se invitó en forma escrita y/o vía internet a participar a los laboratorios clínicos de Lima Metropolitana, por medio de comunicación del Colegio Profesional Tecnólogo Médico del Perú (CTMP), de manera anónima y previo consentimiento informado.

Se envió muestras a 97 laboratorios clínicos, de los cuales 88 laboratorios participaron enviando los resultados. Los laboratorios correspondieron a 25 centros de salud, 16 centros materno infantil, 12 hospitales, 9 laboratorios de EsSalud, 2 de institutos nacionales, 2 laboratorios de referencia, 6 de universidades y 16 laboratorios particulares, ubicados entre los distritos de Lima Cercado, Jesús María, Lince, La Victoria, Comas, Rímac, Ate-Vitarte, San Juan de Miraflores, San Juan de Lurigancho, Los Olivos, Villa el Salvador y de la provincia del Callao.

Como material biológico se usó suero humano fresco de voluntarios, obtenido de manera usual, por puntura de la vena cefálica o basílica del brazo, con ligadura y sistema de extracción al 
Tabla 1. Índice de calidad de la precisión según la variabilidad biológica.

\begin{tabular}{ccccc} 
Índice de calidad & Nivel de calidad & \multicolumn{3}{c}{ Determinación o prueba } \\
\cline { 3 - 5 } Precisión & Glucosa & Colesterol & Triglicéridos \\
Cva $<0,25$ Cvi & Otima & 1,42 CV\% & 1,35 CV\% & 5,23 CV\% \\
Cva $<0,50$ Cvi & Deseable & 2,85 CV\% & 2,70 CV\% & 10,45 CV\% \\
Cva $<0,75$ Cvi & Mínima & 4,27 CV\% & 4,05 CV\% & 15,67 CV\% \\
Cva $>0,75$ Cvi & Fuera & $>4,27$ CV\% & $>4,05$ CV\% & $>15,67$ CV\% \\
\hline
\end{tabular}

Tabla 2. Precisión por coeficiente de variación porcentual para glucosa, colesterol y triglicéridos, de una muestra de laboratorios clínicos de Lima.

\begin{tabular}{cccc} 
CV\% & Glucosa & Colesterol & Triglicéridos \\
Promedio & 3,2 & 5,0 & 5,8 \\
Valor máximo & 14,7 & 40,3 & 31,3 \\
Valor mínimo & 0,0 & 0,0 & 0,0 \\
\hline
\end{tabular}

vacío, sin anticoagulante. Se dejó coagular durante 15 minutos y se obtuvo el suero por centrifugación a $5000 \mathrm{rpm}$ por $5 \mathrm{~min}$. Se colectó el suero de los diferentes tubos en un solo recipiente y fue nuevamente centrifugado para separar posible contaminación hemática durante la colección del suero. Posteriormente, fue alicuotado en crioviales con $0,5 \mathrm{~mL}$ del suero y, rotulados como muestras diferentes -muestra 'A' y muestra 'B'- se los colocó en refrigeración a temperatura de 4 a $8^{\circ} \mathrm{C}$.

Entre 12 a 16 horas después, los crioviales con suero humano fresco 'A' y 'B' fueron distribuidos a los laboratorios participantes en cadena de frío de $4^{\circ}$ a $6^{\circ} \mathrm{C}$, para la determinación de glucosa, colesterol total y triglicéridos en ambas muestras, por métodos enzimático colorimétricos, de acuerdo al protocolo de cada institución. Los laboratorios participantes no tuvieron conocimiento que las muestras eran iguales. Los resultados fueron remitidos electrónicamente, informando la concentración de cada analito y la forma del procedimiento, sea de forma automatizada o manual. Los resultados fueron recibidos desde horas después de la entrega hasta tres días después.

Para la evaluación de la precisión intralaboratorial se utilizó los resultados de concentración emitidos por los laboratorios; se determinó la media, la desviación estándar y el coeficiente de variación porcentual, por medio de las siguientes fórmulas, respectivamente.

$$
\begin{aligned}
& m=\frac{1}{n} \sum_{i=0}^{n-1} x_{i} \\
& \sqrt{s^{2}}=\sqrt{\frac{\sum_{i=1}^{n}\left(x_{i}-\bar{x}\right)^{2}}{n-1}} \\
& C V=\frac{\sqrt{s^{2}}}{m}(100 \%)
\end{aligned}
$$

Para la evaluación de la precisión interlaboratorial, se utilizó el índice de desviación estándar (SDI).

$\mathrm{SDI}=\frac{\text { (media del laboratorio }- \text { media del grupo análogo) }}{\text { Desviación estándar del grupo análogo }}$

El SDI objetivo es 0,0. Esto indica que el desempeño del laboratorio es idéntico al promedio del grupo análogo. Los valores aceptables de SDI están entre $\pm 1,0$. Cualquier prueba/método/ instrumento que tenga un SDI entre $\pm 1,0$ y 1,5 puede tener un problema y el laboratorio debe investigarlo. El laboratorio debe solucionar el problema y corregir cualquier prueba/método/instrumento que tenga un SDI de $\geq 1,5$.

También se valoró la precisión usando la validación de la variabilidad biológica (VB), es decir, la fluctuación fisiológica de los constituyentes de los fluidos orgánicos alrededor de su punto homeostático con sus dos componen(tabla 1). tes: variación intra e interindividual

\section{RESULTADOS}

Se señala los resultados de sueros de 88 crioviales ('A') y 88 crioviales duplicados ('B'), de un total de 97 envíos, alcanzando un total de 528 determinaciones de glucosa, colesterol y triglicéridos.

Todas las pruebas realizadas fueron de tipo enzimático, reacciones acopladas a punto final, colorimétricas y líquidas. La glucosa fue realizada por el método glucosa oxidasa-peroxidasa, el colesterol por colesterol oxidasaperoxidasa, y triglicéridos por método enzimático colorimétrico. Las determinaciones manuales fueron 74 , y 14 por métodos automatizados.

Las pruebas realizadas fueron de tipo enzimático, reacciones acopladas a punto final, colorimétricas y líquidas; la glucosa fue realizada por el método glucosa oxidasa-peroxidasa; para colesterol, colesterol oxidasa-peroxida; y para triglicéridos, método enzimático colorimétrico; 74 de las 88 determinaciones fueron determinadas por métodos manuales y 14 por métodos automatizados.

La precisión observada como el CV\% en promedio fue menor en la determinación de glucosa que en colesterol o triglicéridos, con un intervalo de variación mayor en colesterol hasta 40,3\%, triglicéridos $31,3 \%$ y glucosa $14,7 \%$ (ver tabla 2). Sin embargo, la distribución de la imprecisión mayor al CV 5\% fue para colesterol y triglicéridos.

La evaluación de la precisión metrológica observada mediante índice de desviación estándar (SDI) para glucosa, colesterol y triglicéridos muestra que la mayoría de laboratorios se encuentra en control, sea esta óptima, deseable o mínima; el $42 \%$ de los laboratorios se encontró fuera de control para la prueba del colesterol, $25 \%$ fuera de control para la glucosa y $11,4 \%$ para triglicéridos (tabla 3).

La precisión valorada como CV\%, así como con el SDI tanto para glucosa, colesterol y triglicéridos, siempre fue mejor con el método automatizado que 
con el manual; la dispersión y ubicación de cada laboratorio participante se puede observar en las figuras 2, 3 y 4 para la glucosa, colesterol y triglicéridos, respectivamente. En cada figura, los puntos rojos corresponden a los laboratorios con método de medición automatizada. Hacia el lado derecho se encuentra una franja de colores que señala los límites del SDI. La zona verde $(+/-1)$ es la zona de precisión aceptable, la amarilla $+/-1,5$ zona de revisión y la roja mayor $\mathrm{a}+/-1,5$, fuera de control.

\section{DISCUSIÓN}

Siempre se ha mencionado que el control de calidad en el laboratorio clínico es un procedimiento necesario para la validación de los resultados. Este control puede ser realizado de diversas maneras para la observación de diferentes parámetros de control de calidad. Entre ellos figura la precisión de las mediciones, uno de los procedimientos de control intralaboratorio y que, cuando se compara los resultados de una misma muestra entre varios laboratorios, se transforma en una observación de calidad interlaboratorial. La precisión intralaboratorio se refiere a la concordancia observada entre los resultados obtenidos al analizar varias veces una misma muestra en el mismo laboratorio. La mejor forma de conocerla es mediante pruebas de reproducibilidad ${ }^{(11,12)}$ y se controla mediante métodos de control de calidad interno ${ }^{(13,14)}$. Es por ello que nuestro estudio refleja la precisión, resaltando además que esta ha sido de manera ciega, ya que los laboratorios participantes no fueron informados que las muestras enviadas para su procesamiento tenían la misma procedencia, es decir del mismo donante, hecho que valida el procedimiento y fidelidad de los resultados. $\mathrm{Al}$ ser las dos muestras enviadas de un solo donante o una sola procedencia, los resultados producto del análisis en teoría deberían ser iguales. Pero, esto realmente es muy poco probable, ya que en las mediciones siempre hay oscilaciones. Lo conveniente es que estas variaciones sean
Tabla 3. Precisión metrológica mediante el índice de desviación estándar (SDI) para glucosa, colesterol y triglicéridos.

\begin{tabular}{|c|c|c|c|c|c|c|c|}
\hline \multirow{2}{*}{$\begin{array}{c}\text { Índice } \\
\text { SDI }\end{array}$} & \multirow{2}{*}{$\begin{array}{c}\text { Resultado } \\
\text { Valores }\end{array}$} & \multicolumn{2}{|c|}{ Glucosa } & \multicolumn{2}{|c|}{ Colesterol } & \multicolumn{2}{|c|}{ Triglicéridos } \\
\hline & & $\mathrm{n}$ & $\%$ & $\mathrm{n}$ & $\%$ & $\mathrm{n}$ & $\%$ \\
\hline Aceptable & Entre +/- 1 & 70 & 79,5 & 70 & 79,5 & 64 & 72,7 \\
\hline Revisión & Entre $+/-1,5$ & 10 & 11,4 & 8 & 9,1 & 13 & 14,8 \\
\hline Fuera de rango & Mayor $+/-1,5$ & 8 & 9,1 & 10 & 11,4 & 11 & 12,5 \\
\hline Total & & 88 & 100,0 & 88 & 100,0 & 88 & 100,0 \\
\hline
\end{tabular}

mínimas dentro de los rangos que son aceptados como dentro de control.

En nuestra investigación, para el caso de glucosa, la variación máxima del CV fue $14,7 \%$ (ver tabla 2), lo que significa que una persona sana con 90 $\mathrm{mg} / \mathrm{dL}$ puede tener el informe de una glicemia de $110 \mathrm{mg} / \mathrm{dL}$, como lo fue en nuestro estudio; o en un caso de algún paciente con diabetes, dicho laboratorio podría informar de la misma muestra una variación entre 130 y $148 \mathrm{mg} /$ dL de glucosa. Para el caso del colesterol, el CV\% máximo en un laboratorio fue 40,3\% (tabla 2), lo que significaría que si un paciente tiene $180 \mathrm{mg} / \mathrm{dL}$ de colesterol -valor considerado como normal-, dicho laboratorio informaría aleatoriamente hasta $257 \mathrm{mg} / \mathrm{dL}$; y, para el caso de triglicéridos, dicha variación máxima observada fue de 31,3\%, por lo que si un paciente tuviera $240 \mathrm{mg} /$ dL de triglicéridos séricos (valor por encima del normal) el laboratorio podría generar un resultado de $182 \mathrm{mg} / \mathrm{dL}$. Estos ejemplos muestran la variación encontrada en los resultados emitidos entre los laboratorios participantes y evidentemente la variación influiría en la toma de decisiones médico-clínicas, con diagnósticos y procedimientos tal vez errados. Debemos resaltar que fueron los valores máximos de imprecisión alcanzados. Más adelante discutiremos fueron encontrados en condiciones de control.

Según señalan Vargas y cols. ${ }^{(15)}$, en Costa Rica, los laboratorios que no realizan control de calidad interno no cuentan con una definición de variabilidad máxima permisible, de acuerdo con los métodos, equipo y condiciones de trabajo de los laboratorios costarricenses. Usualmente, los laboratorios determinan que existe un error cuando un resultado excede el promedio $(\mathrm{X}) \pm$ $2 \mathrm{DE}$, lo cual no es válido cuando las DE son amplias ${ }^{(16)}$, práctica que también se realizó en la muestra estudiada. La validación de la precisión de los procedimientos de medida también es un requisito de las normas internacionales utilizadas para implantar un sistema de gestión de la calidad en los laboratorios clínicos (ISO 9001: 2000, ISO 15189: 2003) ${ }^{(17)}$.

Al observar los resultados del indicador SDI, se puede apreciar que sus valores oscilaron entre 9 y $12 \%$ para los tres parámetros medidos, que aparentan que la situación de calidad no la proporción de laboratorios que no

Tabla 4. Evaluación del desempeño metrológico del índice de calidad de precisión mediante la variabilidad biológica para glucosa, colesterol y triglicéridos.

\begin{tabular}{|c|c|c|c|c|c|c|c|}
\hline \multirow{2}{*}{$\begin{array}{l}\text { Índice de calidad } \\
\text { Precisión }\end{array}$} & \multirow{2}{*}{$\begin{array}{l}\text { Nivel de } \\
\text { Calidad }\end{array}$} & \multicolumn{2}{|c|}{ Glucosa } & \multicolumn{2}{|c|}{ Colesterol } & \multicolumn{2}{|c|}{ Triglicéridos } \\
\hline & & $n$ & $\%$ & $\mathrm{n}$ & $\%$ & $\mathrm{n}$ & $\%$ \\
\hline $\mathrm{CVa}<0,25 \mathrm{CVi}$ & Óptima & 36 & 40,9 & 27 & 30,7 & 58 & 65,9 \\
\hline $\mathrm{CVa}<0,50 \mathrm{CVi}$ & Deseable & 16 & 18,2 & 17 & 19,3 & 14 & 15,9 \\
\hline $\mathrm{CVa}<0,75 \mathrm{CVi}$ & Mínima & 14 & 15,9 & 7 & 8,0 & 6 & 6,8 \\
\hline CVa $>0,75 \mathrm{CVi}$ & Fuera & 22 & 25,0 & 37 & 42,0 & 10 & 11,4 \\
\hline Total & & 88 & 100,0 & 88 & 100,0 & 88 & 100,0 \\
\hline
\end{tabular}




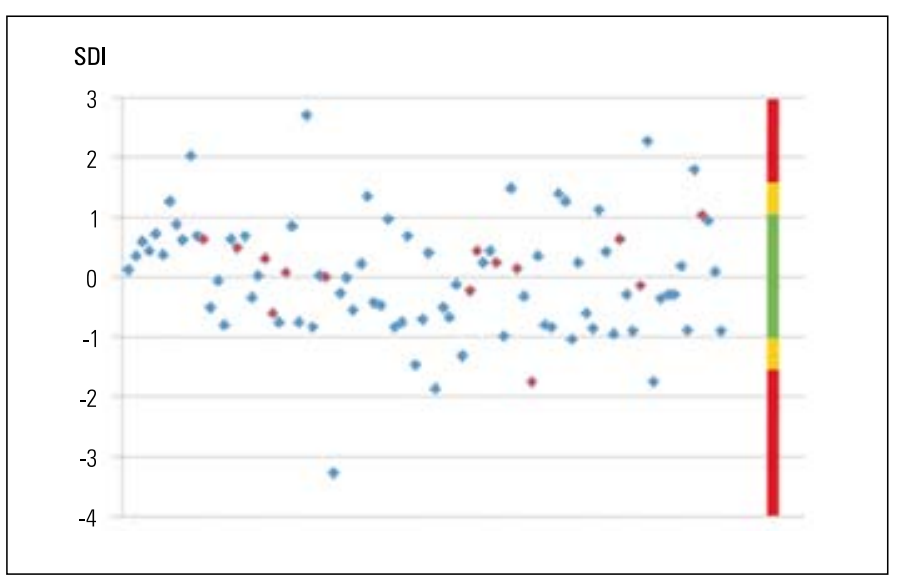

Figura 2. Índice de desviación estándar (SDI) para glucosa. Método manual = puntos azules, automatizado $=$ puntos rojos. La zona verde $(+/-1$ del SDI) es la zona de precisión aceptable, la amarilla de revisión y la roja fuera de control. Los puntos rojos corresponden a los laboratorios con procedimiento automatizado.

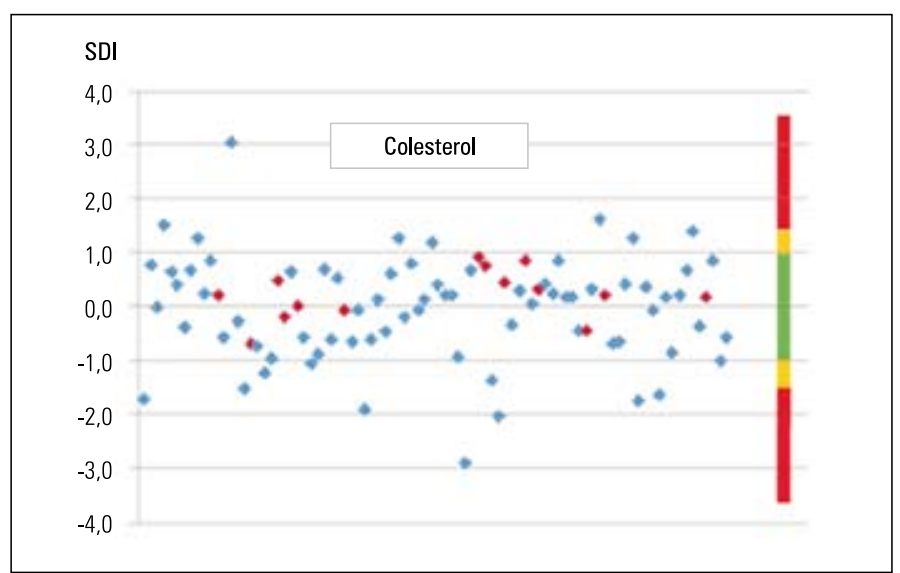

Figura 3. Índice de desviación estándar (SDI) para colesterol. Método manual = puntos azules; automatizado = puntos rojos. La zona verde $(+/-1$ del SDI) es la zona de precisión aceptable, la amarilla de revisión y la roja fuera de control. Los puntos rojos corresponden a los laboratorios con procedimiento automatizado.

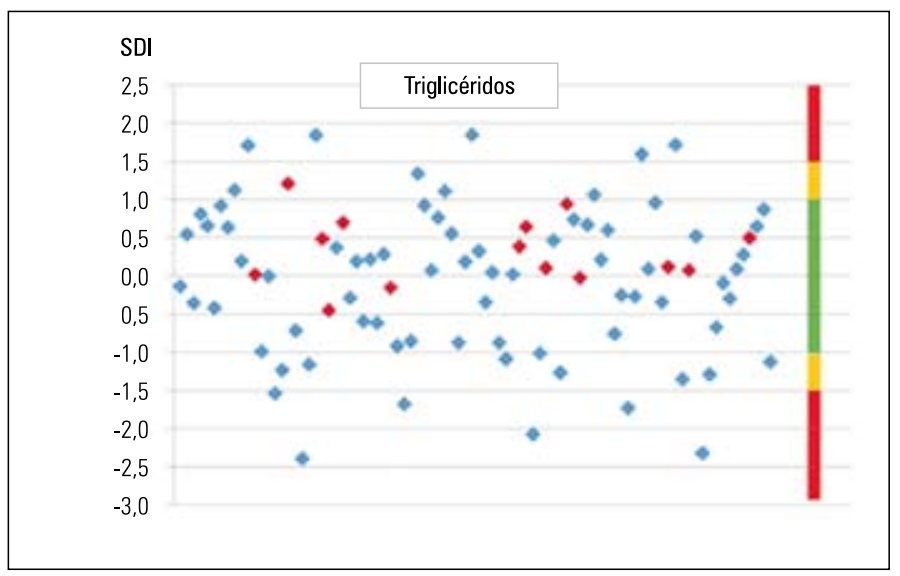

Figura 4. Índice de desviación estándar (SDI) para triglicéridos. Método manual = puntos azules; automatizado $=$ puntos rojos. La zona verde $(+/-1$ del SDI) es la zona de precisión aceptable, la amarilla de revisión y la roja fuera de control. Los puntos rojos corresponden a los laboratorios con procedimiento automatizado. es la óptima. Además, se puede denotar que entre los laboratorios participantes, aquellos que tienen equipos totalmente automatizados fueron los que mejor precisión tuvieron y fueron los laboratorios con método manual los que tuvieron mayores variaciones. Se evidencia que la automatización es un potencial que se puede usar para colesterol, glucosa y triglicéridos, como se puede observar en las figuras 2, 3 y 4) al visualizar los resultados de los laboratorios automatizados, en color rojo.

También, debemos señalar que hubo laboratorios con procedimiento manual que presentaron valores muy aceptables y menores a las dos desviaciones estándar. De manera semejante, cumplieron con los estándares de control de calidad al estar dentro de los márgenes de variabilidad biológica aceptables, lo cual denotaría que el procesamiento manual en el laboratorio clínico es también posible. Sabemos que no son únicamente los equipos que entran como variables en los resultados finales; están además el tratamiento de la muestra después de recibida en el laboratorio, la experticia del operador, calidad de los reactivos, entre otras variables. En las diferentes figuras se puede evidenciar la mayoría de estas características.

La evaluación de precisión metrológica mediante el índice de desviación estándar (SDI) observa el grado de dispersión del conjunto de laboratorios, es decir, se transforma en un parámetro de observación interlaboratorial. La estimación de fuera o dentro de control se refiere a la proximidad del valor del laboratorio observado respecto a la media interlaboratorial obtenida. Así, hemos encontrado que para glucosa el 9,1\% de laboratorios estuvo fuera de rango estadísticamente aceptable; para colesterol lo fue el 11,4\% de los laboratorios participantes, y para triglicéridos el $12,5 \%$.

Dentro de los errores frecuentes que los laboratorios clínicos refieren, son los que se encuentran en la parte preanalítica y, entre ellos, los causados por los mismos pacientes, ya sea en el incum- 
plimiento de las especificaciones de recogida de la muestra y las condiciones fisiológicas; estos errores son aproximadamente $73 \%$ y son responsables del $24 \%$ de ensayos repetidos ${ }^{(18)}$, hecho que en nuestra investigación no se dio, puesto que se trató de un solo pool de sueros, homogenizados y dispensados, por lo que los resultados observados resultan de la variación intralaboratorial de cada uno de los laboratorios que participaron de la muestra.

El control de la calidad en el laboratorio clínico es interno como externo. Otras experiencias muestran un número de eventos de control externo semejante al desarrollado por nuestro estudio, en un número de 4 hasta 20 veces, todo ello en pos de asegurar la calidad de los resultados de los diversos análisis procesados en el laboratorio clínico; siendo pues necesario un sistema de control de calidad externo o interlaboratorial ${ }^{(19)}$. En nuestro país existe la norma que regula el control de la calidad en los laboratorios clínicos (NTS 072); sin embargo, creemos que no se encuentra totalmente socializada entre el personal de salud y consecuentemente no es aplicada aún.

Los resultados del laboratorio clínico son importantes, pues son utilizados en muchas situaciones clínicas relevantes, que incluyen el diagnóstico, tratamiento, pronóstico, detección de cambios metabólicos; también, sirven como fuente de investigación, desarrollo, enseñanza y formación, y puede ser que una sola forma de control de la calidad no sería apropiada para todos ellos. Sin embargo, algunos autores señalan que ni médicos ni pacientes han sufrido daño por los resultados de los análisis de laboratorio, puesto que el médico se vale de la semiología clínica. Pero, con la diversidad de datos provenientes de los exámenes auxiliares, cada vez con mayor sensibilidad y especificidad, el laboratorio se ha constituido en una fuente de información del estado metabólico del paciente y de allí la importancia y el empeño de los fabricantes de sistemas de análisis, reactivos y equipos con tecnología de punta ${ }^{(20)}$.
Finalmente, puede decirse que en la muestra de 88 laboratorios participantes, la mayoría tuvo una buena precisión, dentro de los parámetros de oscilación considerados como de calidad. Sin embargo, todavía un 25\% de laboratorios para la determinación de glucosa, $42 \%$ para colesterol y $11,4 \%$ para triglicéridos se encuentran fuera del rango de control, con la valoración de la precisión observada con la variabilidad biológica. Estos resultados pueden explicar de alguna manera la desconfianza que se genera en algunas ocasiones al tener resultados diferentes en un mismo paciente o de una misma muestra en dos instituciones diferentes. Esta experiencia aquí mostrada no es para desarrollar una corriente de opinión desfavorable sino para observar la realidad y cómo es la razón del control de calidad, ofrecer oportunidad de mejora, en pos de la satisfacción de los usuarios y lograr tener un mayor y acertado apoyo al diagnóstico, pronóstico y tratamiento de las patologías.

En conclusión, la precisión en la mayoría de los laboratorios clínicos de Lima, participantes en el estudio, fue aceptable o dentro de control (entre 70 y 75\%). Los resultados de los valores de glucosa, colesterol total y triglicéridos séricos de los laboratorios con sistema de medición automatizada, presentaron siempre mejor precisión. Sin embargo, aún existen laboratorios con imprecisión en sus resultados, por lo que se debe hacer esfuerzos para mejorar estos índices de calidad.

\section{AGRADECIMIENTOS}

A los 88 laboratorios clínicos de Lima y su personal, que anónimamente aceptaron participar.

Al Colegio Tecnólogo Médico del Perú, en la persona del Lic. TM. Carlos Penalillo Pimentel.

\section{REFERENCIAS BIBLIOGRÁFICAS}

1. Sáez S, Pastor L, Alvariño A. Control externo de calidad: comparación de dos métodos de evaluación. Sociedad
Española de Dirección y Gestión de los Laboratorios Clínicos. VIII Reunión Vigo, 10-12 de marzo de 2004. Comunicación -36.

2. Ferrucio C. El concepto de calidad en los laboratorios clínicos. Acta Bioq Clín Latinoam. 2000;34(3):392-4

3. Armendáriz $Y$, Ruigómez $P$, Dot D, Ricós $C$, Galimany R. Influencia de los programas de evaluación externa de la calidad en la estandarización de las determinaciones enzimáticas. Quim Clin. 2002;21(6):454-9.

4. Garcia-Lario J, Álvarez V, Cava F, Hernández A, Jiménez C. Aplicabilidad de los datos de variabilidad biológica. I. Especificaciones de la calidad analítica. Quim Clin. 2001;20(6):450-6.

5. Ramón F, Alsina MJ, Álvarez V, Cortés M. XXII Programa de Evaluación Externa de la Calidad de Bioquímica Clínica y Patología Molecular. Quim Clin. 2002;21(3):93151

6. Ricós C. Objetivos de la calidad analítica del laboratorio clínico. Barcelona. Scand J Clin Lab Invest. 1999;59(7).

7. Ministerio de Salud. Norma técnica de salud $N^{\circ} 072$, de la unidad productora de servicios de patología clínica. NTS No 072 - MINSA/DGSP - V.01. Resolución Ministeria № 627-2008/MINSA

8. Marianela U, Jessie M, Denis A, Schosinsky N. Precisión intralaboratorio en química clínica según el control de calidad interno en un grupo de laboratorios costarricenses. Disponible en: http://www.binasss.sa.cr/revistas/ $\mathrm{rccm} / \mathrm{v} 10 \mathrm{n} 4 /$ art6.pdf

9. Vocabulaire international de métrologie - Concepts fondamentaux et généraux et termes associés (VIM) Disponible en : http://www.ume.tubitak.gov.tr/duyurular/ Uluslararasi_Metroloji_Sozlugu.pdf

10. Nury G. Control y Garantía de Calidad en Laboratorios Analíticos. Disponible en: http://web.usach.cl/ima/ ngras.htm

11. Dharán M. Control de calidad en los laboratorios Clínicos. Barcelona: Editorial Reverté S.A. 2002.

12. Zhichu C. Allowable limit of error in Clinical. Chemistry Quality Control. Clin Chem. 1988;35(4):630-1.

13. Peters T, Westgard J. Evaluation of Clinical Chemistry Philadelphia: W.B. Saunders Company, 1987:225-37.

14. Westgard J, Klee G. Quality assurance. En: Tietz NW, ed. Textbook of Clinical Chemistry. Philadelphia: W.B. Saunders Company. 1986:424-57.

15. Vargas M, Orlich J, León D, Schosinsky K. Precisión intralaboratorio en química clínica según el control de calidad interno en un grupo de laboratorios costarricenses. Departamento de Análisis Clínicos y CIHATA, Universidad de Costa Rica. Publicaciones del Cihata, 1982-1988.

16. Zhichu C. Allowable limit of error in clinical chemistry quality control. Clin Chem. 1988:35(4):630-1

17. Comité Europeo de Normalización. Medical laboratorios - Particular requirements for quality and competence. EN ISO 15189; 2003. Bruselas: CEN.

18. Carraro P, Plebani M. Errors in a stat laboratory: types and frequencies 10 years later. Clin Chem. 2007:53(7):1338-42.

19. Thomas A. External quality assessment in laboratory medicine: is there a rationale to determine frequency of surveys? Accred Qual Assur. 2009:14:439-44.

20. Petersen $\mathrm{H}$, Fraser $\mathrm{C}$. Strategies to set global analytical quality specifications in laboratory medicine: 10 years on from the Stockholm Consensus Conference. Accred Qual Assur. 2010;15:323-30.

\section{Financiamiento}

Proyecto Calidad Metrológica, financiado por el Consejo Superior de Investigaciones de la Universidad Nacional Mayor de San Marcos.

\section{Conflictos de interés:}

Los autores no tuvieron conficto de interes por no haber participado en los procesos laboratoriales de las determinaciones observadas en el estudio.

\section{Correspondencia:}

mhsave@gmail.com 